\title{
Whispering Gallery Mode Emission from Photonic Microtubes
}

\author{
John F. Donegan ${ }^{1 *}$, Member, IEEE, Yury Rakovich ${ }^{1}$, Sivakumar Balakrishnan ${ }^{2}$,Yurii Gun'ko ${ }^{2}$ \\ Tatiana Perova ${ }^{3}$, and Alan Moore ${ }^{3}$ \\ ${ }^{1}$ Semiconductor Photonics Group, School of Physics, Trinity College Dublin, Ireland \\ ${ }^{2}$ School of Chemistry, Trinity College Dublin, Ireland \\ ${ }^{3}$ Department of Electronic and Electrical Engineering, Trinity College Dublin, Ireland \\ *Tel: +353-1-6081987, Fax: +353-1-6711759, e-mail: jdonegan@tcd.ie
}

\begin{abstract}
We have found cylindrical glass microtubes can be formed by vacuum assisted filtration using a micro-channel glass matrix followed by thermal treatment of the material. The microtubes are strongly luminescent and exhibit sharp whispering gallery modes. The ease of fabrication suggest that such photonic structures will find use as both microsensors and microlasers.
\end{abstract}

Keywords: microtubes, whispering gallery modes, photonic structures, microlasers.

\section{INTRODUCTION}

The fabrication of tubular materials have attracted lots of attention in recent years and many reports have dealt with the syntheses of tubular materials such as silica, magnesium oxide, titanium dioxide and zinc oxide. [1] The fabrication of micro-wire arrays in situ using electrochemical [2-4] and electroless [5-7] deposition in a porous template has proven to be a superior option for preparing more complex, higher aspect ratio 3-D $\mu$-tube and $\mu$-wire arrays.[8] However, this method of preparation can be expensive and tedious.

In this paper, we describe an easy method for fabricating luminescent aluminosilicate xerogel microtubes and their optical characterisation. We have studied the structure of whispering gallery modes in the photoluminescence (PL) spectra of microtubes under different excitation conditions across a wide spectral region. Such microtube resonators are proposed as a versatile building block suitable for integration in communication curcuits due to their size, spectral and material compatibility with optical fiber. Moreover, the high dispersion per unit volume can be provided by increasing the circulating intensities in the microtubes yet the microcavities themselves can be fabricated with conventional processing techniques which do not require either cutting-edge technology or exotic materials.

\section{EXPERIMENTAL DETAILS}

Micro-channel glasses were cleaned using acetone and dried. Then the micro-channel glass sample was placed in the filtration chamber and a vacuum of 29-30 mbar was slowly applied. The "sol", just before its gelation point was placed onto these micro-channel glass samples and vacuum assisted filtration resulted in the formation of fibres inside the micro-channel glass matrix. Then the composite was dried at room temperature for 1 day and further annealed at $500^{\circ} \mathrm{C}$ for 2 hours. Finally, the micro-tubes were drawn from the matrix by a simply cutting the matrix.

The steady-state PL spectra from a single microtube were recorded in a backscattering geometry using a RENISHAW micro-Raman system (1800 mm-1 grating, $>1 \mathrm{~cm}^{-1}$ resolution) equipped with a microscope objective $(\times 100)$, a notch and plasma filters and a CCD camera. An Ar+ laser (wavelength $514.5 \mathrm{~nm}$ ) was used in the micro-PL measurements.

PL decays were measured using time-correlated single photon counting (Time-Harp, PicoQuant). The samples were excited by $480 \mathrm{~nm}$ picosecond pulses at a $20 \mathrm{MHz}$ repetition rate with an overall time resolution of $\sim 150$ psec. Decays were measured to 6000-8000 counts in the peak and reconvoluted using non-linear least squares analysis (FluoFit, PicoQuant), using an equation of the form: $I(t) \propto \sum_{i} \alpha_{i} \exp \left(-t / \tau_{i}\right)$, where $\tau_{\mathrm{i}}$ are the

PL decay times. The pre-exponential factors $\alpha_{i}$, were taken into account by normalisation of the initial point in the decay to unity.

\section{RESULTS AND DISCUSSION}

The as-prepared aluminosilicate solgel-microchannel glass composite was annealed at $500^{\circ} \mathrm{C}$ for $2 \mathrm{hrs,} \mathrm{the}$ microtubes have an outer diameter of $6-7 \mu \mathrm{m}$ and the inner diameter of $2.7-3 \mu \mathrm{m}$.

A typical PL spectrum of single aluminosilicate microtube accommodated in micropore is presented in Fig. 1a. The emission spectra shows a broad luminescence ranging between 520 and $800 \mathrm{~nm}$. The probable origin of the emission comes is the organic species trapped inside the gel matrix might that have decomposed

The work reported in this paper was supported in part under the CRANN CSET Project PR03 Photonic Molecules and in part under the grant EI. 
and created a carbon substitutional defect for silicon resulting in strong luminescence in the thermally treated gels.
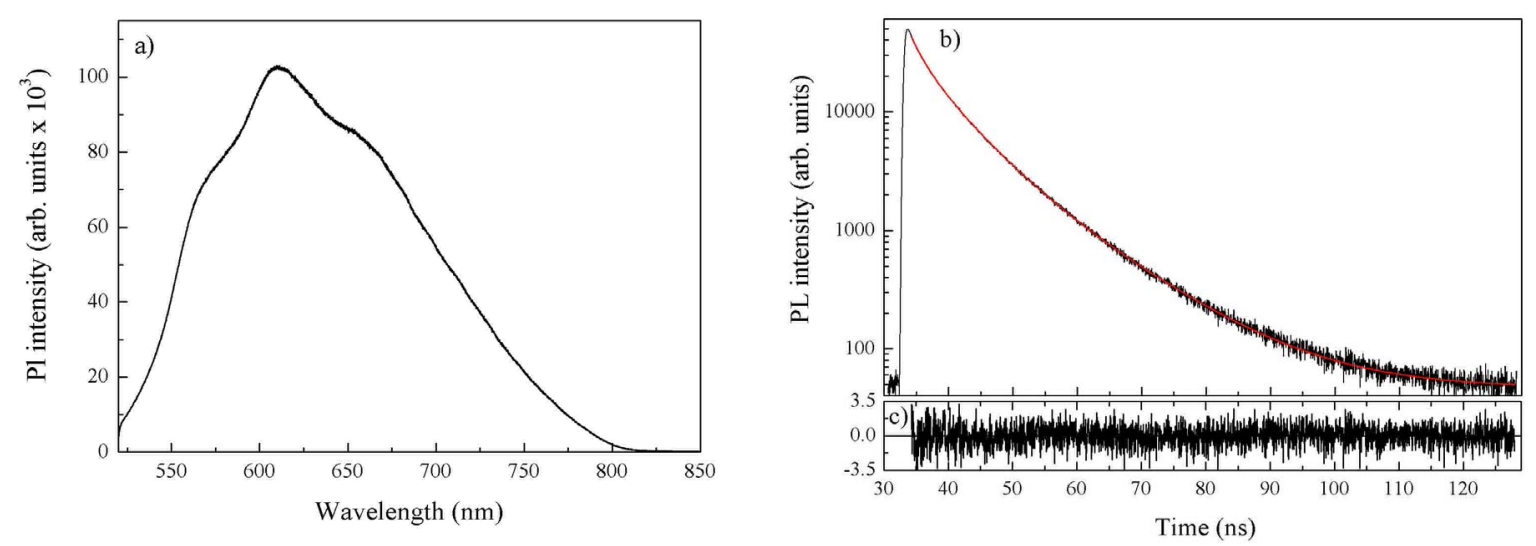

Fig. 1. PL spectra (a) and time-dependent PL intensity decay of a single aluminosilicate microtube accommodated in a glass micropore (b). Results of three-exponential analysis of decay curves are shown by thick red line with corresponding residual (c).

The PL of a single aluminosilicate microtube shows distinct multi-exponential decay. The Sum of at least threeexponential functions is required to achieve satisfactory fit to the decay data (Fig. 1b) yielding a reasonable plot of weighted residuals (Fig. 1c). The observed multi-exponential decay kinetics are consistent with a PL model that includes multiple emission pathways and is indicative of broad lifetime distribution caused by structural heterogeneity of the glass and a corresponding distribution of defect or trap states.

In contrast to the broad, featureless PL band in the spectra of tubes embedded in a micro-porous glass matrix (Fig. 1a), the emission spectra of a single "free standing" microtube exhibit very sharp periodic structure (Fig. 2). The observed peaks are the so-called whispering-gallery mode (WGM) resonances: a result of coupling of electronic states in aluminosilicate gel and photon states of the microtube.

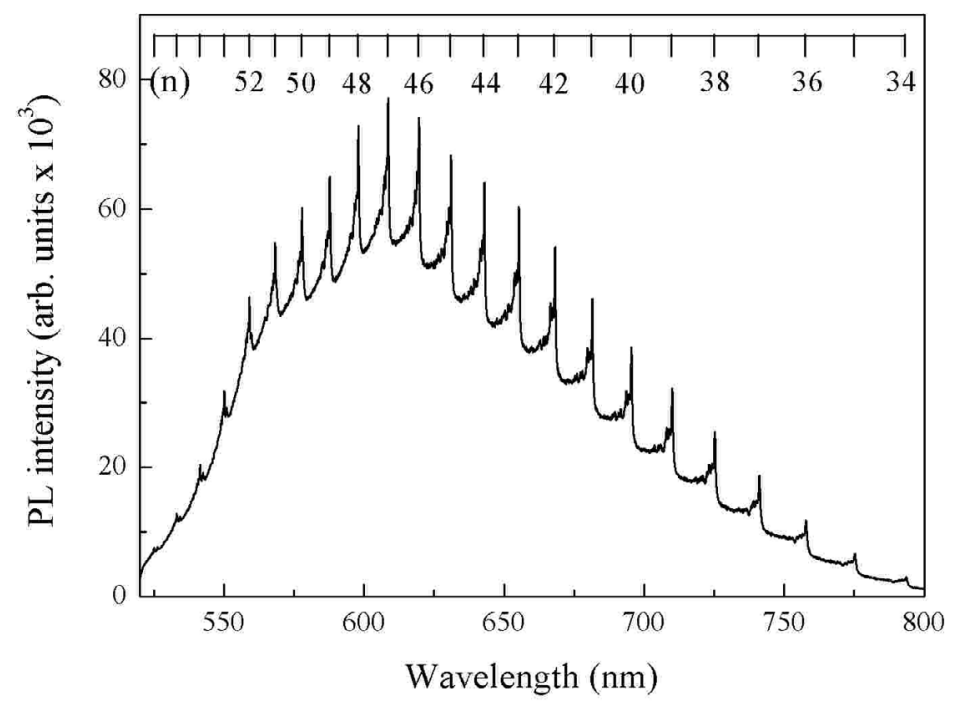

Figure 2. PL spectra of a single aluminosilicate microtube. Result of WGM identification are depicted on the top. The excitation laser beam is polarized parallel to the axis of the microtube.

When embedded into the matrix, the spectral distribution of emission and the individual transition probabilities are almost independent of spatial location due to low refractive index contrast between tube and the matrix. Contrastingly, when separated from the matrix, the microtube is much more optically dense than its surrounding medium, providing that light in the microcavity can be internally reflected. In this way, light propagating inside the microtube would then be spatially constrained to travel along the rim of a crossection of the tube, and therefore it is said to be trapped in a WGM. An immediate result of this optical confinement is the presence of emission peaks in luminescence spectra from single microtubes, as illustrated in Figure 2 . These peaks correspond to optical resonance locations (or WGM) and reflect the fact that transition probabilities are increased for emission wavelengths near resonances. The numbers in figure 2 refer to the mode number as calculated using a Lorentz-Mie theory. 
For a fixed polarizer orientation parallel to the axis of the microtube and the same polarization of excitation beam, the highest intensity in the emission spectra was obtained with peaks located as presented in Figure 3 (top curve). Rotation of the polarizer by $90^{\circ}$ results in strong quenching of this WGM (see bottom curve in Figure 3 ) unambiguously indicating their TM character.

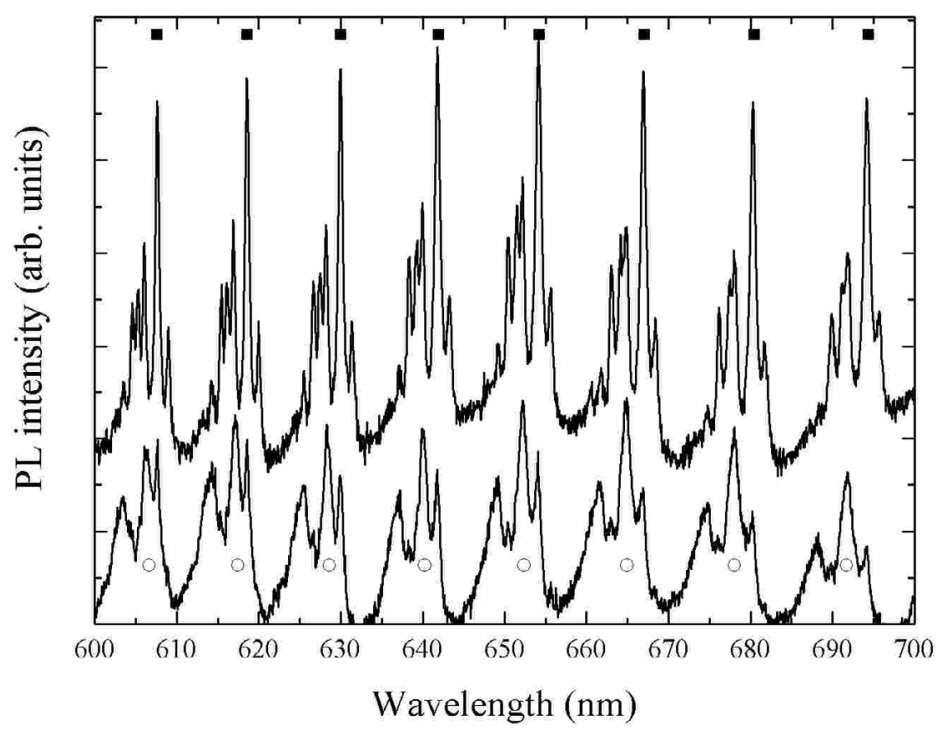

Figure 3. Determination of cavity modes polarization by combining polarization-selective spectroscopy and calculation based on Mie theory. Background PL has been subtracted in the spectra presented in order to show more clearly the WGM structure. Top curve was obtained with a polarizer orientation parallel to the axis of the microtube, whereas the bottom one was detected with the polarizer rotated by $90^{\circ}$. Calculated positions of TM and TE WGM are indicated by squares and circles, respectively.

Nonlinear behaviour of $Q$ can be seen in figure 3: the initial growth of $Q$ value with decreasing wavelength is followed by rapid decrease in the short-wavelength spectral region. This behaviour is known for small spherical microcavities [8] and, in our case can be explained by stronger coupling of the emission to WGM of the microcylinder in the spectral region where the maximum PL is observed. These results suggest that microtube cavities provide a unique possibility of engineering the distribution of photon storage times in a desired spectral region. This controllable delay of an optical signal is a highly desirable functionality for communication signal processing applications.

It is well known that the resonant internal field of a microcavity is not completely confined to the interior of the microresonator. Depending on the shape and size of the microcavity, the evanescent field can extend into the surroundings up to a couple of micrometers. It was recently recognized that the partial delocalization of Mie resonance states is of great importance because it suggests a possibility for coherent coupling between WGMs of two adjacent microcavities with closely matched sizes.[9, 10] For both TE and TM polarizations, it was theoretically shown that the contribution of a resonant mode to the field outside the microcylinder decays exponentially with faster decay predicted for the lower value of mode order $l$. [11] In the present work we experimentally examine the distribution of this evanescent field proceeding from the assumption that tightly focused laser beam passing outside the cavity but within the evanescent tail will preferentially excite specific cavity modes depending on its distance from microtube axis. Due to highly localized nature of WGM of different mode number and/or mode order, the characterization and manipulation of the strength of individual peaks can be achieved.

In Figure 4, the integrated PL efficiency and spectra from different spatial locations of excitation are shown. It is clearly demonstrated that the microcavity effects observed in the microtube are highly dependent on the spatial location of the excitation spot. Not only the integral PL intensity and the ratio between WGM peaks and background emission change with the excitation position, but also the relative strength of different WGM.

These results clearly demonstrate that the strength and efficiency of the WGM are highly dependent on the excitation location. The evanescent field associated with cavity modes extends into the surroundings up to a distance comparable to the external radius of the microtube. This observation along with selective control of WGM visibility suggest a possibility of achieving strong evanescent coupling between adjacent high- $Q$ microresonators, and therefore, implies manifold applications, in optical buffering and controllable delay lines as well as in the fields of quantum cryptography, quantum computing, and quantum information processing. It should be particularly emphasized that the multi-peak structure observed in PL spectra of single aluminosilicate microtube is governed by optical, but not by material parameters of the micocavity and would 
not be radically altered in scattering spectra, lasing, Raman or stimulated Raman scattering in the same way that in a semiconductor laser the lasing does not alter the cavity resonances.
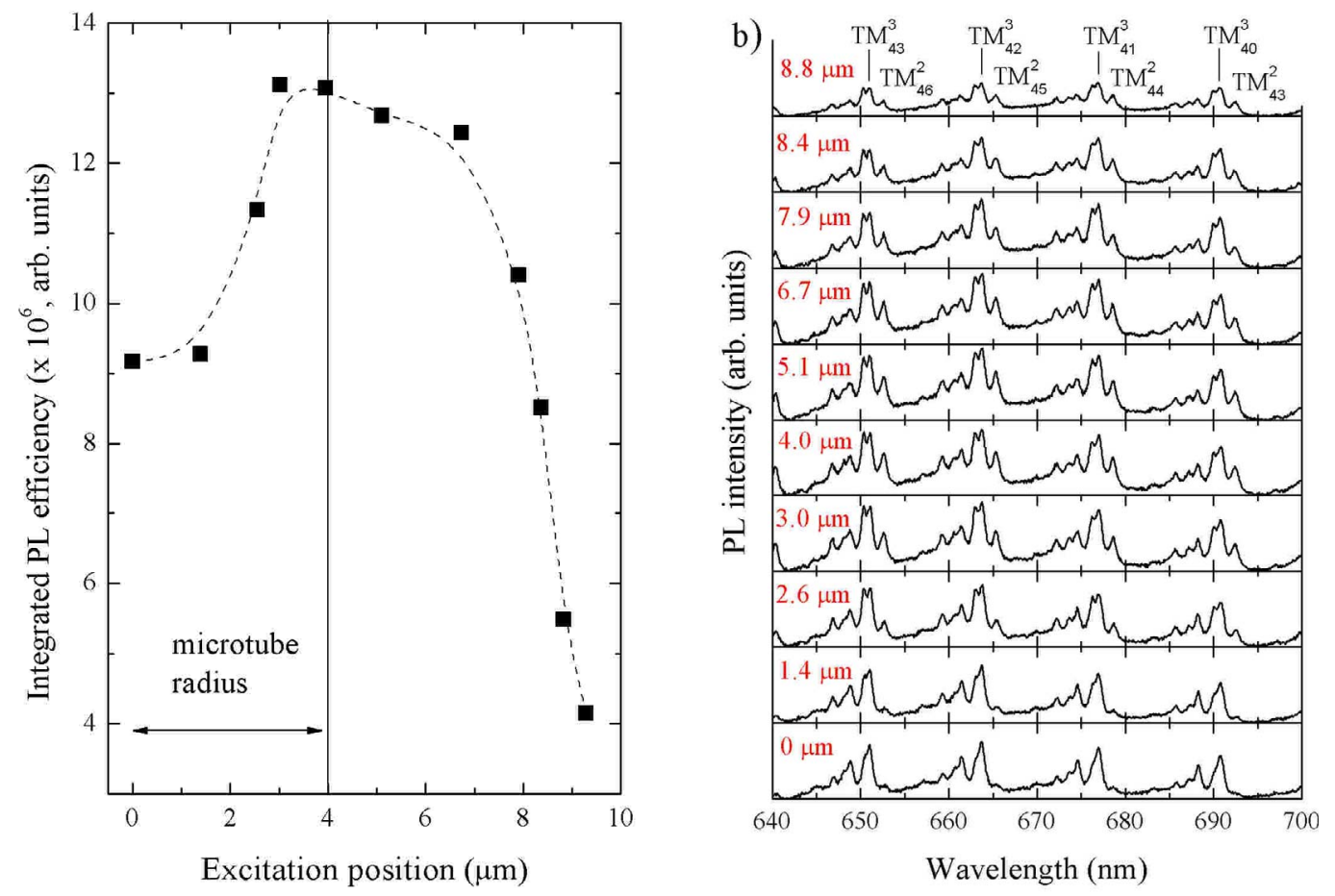

Figure 4. (a) Total luminescence intensity versus excitation beam position in single microtube. Position $0 \mu \mathrm{m}$ corresponds to the excitation point located on the axis of microtube. (b) Emission spectra for different locations of excitation spot. Vertical scale is the same for all panels. Background PL has been subtracted in all spectra presented.

\section{CONCLUSIONS}

To summarize, we have developed a simple and robust method for fabricating luminescent aluminosilicate xerogel microtubes and demonstrated the high-Q resonance modes in emission spectra of a single microcavity. The method of preparation of such structures has unique advantages providing high-quality three-dimensional microcavities with strongly polarized emission. The evanescent field associated with cavity modes was found to extend into the surroundings up to a distance comparable to the external radius of the microtube. Potentially these microcavities should be useful for a variety of photonic applications.

\section{REFERENCES}

[1] K. Mitsuhashi, et al:: Synthesis of Microtubes with a Surface of "House of Cards" Structure via Needlelike Particles and Control of Their Pore Size, Langmuir, 2005. 21(8): pp. 3659-3663.

[2] R.M. Penner, and C.R. Martin: Preparation and electrochemical characterization of ultramicroelectrode ensembles, Anal. Chem., 1987. 59(21): pp. 2625-2630.

[3] J. Choi, et al.: Hexagonally Arranged Monodisperse Silver Nanowires with Adjustable Diameter and High Aspect Ratio, Chem. Mater., 2003. 15(3): pp. 776-779.

[4] C.D. Merritt and B.L. Justus: Fabrication of Microelectrode Arrays Having High-Aspect-Ratio Microwires, Chem. Mater., 2003. 15(13): pp. 2520-2526.

[5] S.A. Sapp, D.T. Mitchell and C.R. Martin: Using Template-Synthesized Micro- and Nanowires as Building Blocks for Self-Assembly of Supramolecular Architectures, Chem. Mater., 1999. 11(5): pp. 1183-1185.

[6] Y.L. Tai and H. Teng: Template Synthesis and Electrochemical Characterization of Nickel-Based Tubule Electrode Arrays, Chem. Mater., 2004. 16(2): pp. 338-342.

[7] M. Wirtz and C.R. Martin: Template-Fabricated Gold Nanowires and Nanotubes, Adv. Mater., 2003. 15(5): pp. $455-458$.

[8] Y.P. Rakovich, et al:: Whispering Gallery Mode Emission from a Composite System of CdTe Nanocrystals and a Spherical Microcavity, Sem. Sci. Techn., 2003. 18(11): pp. 914-918.

[9] S. Deng, W. Cai, and V. Astratov: Numerical study of light propagation via whispering gallery modes in microcylinder coupled resonator optical waveguides, Opt. Express, 2004. 12(26): pp. 6468-6480.

[10] Y.P. Rakovich, et al.: Fine structure of coupled optical modes in photonic molecules, Phys. Rev. A, 2004. 70(5): pp. 051801(R)-1/4.

[11] J.F. Owen, R.K. Chang and P.W. Barber: Internal electric field distributions of a dielectric cylinder at resonance wavelengths, Opt. Lett., 1981. 6(11): pp. 540-542. 